\title{
Prognostic effect of class III $\beta$-tubulin and Topoisomerase-II in patients with advanced thymic carcinoma who received combination chemotherapy, including taxanes or topoisomerase-II inhibitors
}

\author{
YOSUKE MIURA $^{1,2}$, KYOICHI KAIRA $^{2,3}$, REIKO SAKURAI ${ }^{4}$, \\ HISAO IMAI $^{1}$, YOSHIO TOMIZAWA ${ }^{4}$, NORIAKI SUNAGA ${ }^{2,5}$, KOICHI MINATO ${ }^{1}$, \\ TAKESHI HISADA $^{2}$, TETSUNARI OYAMA ${ }^{6}$ and MASANOBU YAMADA ${ }^{2}$ \\ ${ }^{1}$ Division of Respiratory Medicine, Gunma Prefectural Cancer Center, Ota, Gunma 373-8550; \\ Departments of ${ }^{2}$ Medicine and Molecular Science, and ${ }^{3}$ Oncology Clinical Development, \\ Gunma University Graduate School of Medicine, Maebashi, Gunma 371-8511; ${ }^{4}$ Division of Respiratory Medicine, \\ National Hospital Organization Nishigunma Hospital, Shibukawa, Gunma 377-8511; 5 Oncology Center, \\ Gunma University Hospital; ${ }^{6}$ Department of Diagnostic Pathology, Gunma University Graduate School of Medicine, \\ Maebashi, Gunma 371-8511, Japan
}

Received March 29, 2016; Accepted January 31, 2017

DOI: $10.3892 / \mathrm{ol} .2017 .6419$

\begin{abstract}
Class III $\beta$-tubulin (TUBB3) and Topoisomerase-II (topo-II) are considered to be the predictors of therapeutic efficacy and outcome in several types of human neoplasm. However, whether TUBB3 or topo-II may predict the response to combination chemotherapy and prognosis in patients with advanced thymic carcinoma (ATC) remains unclear. The aim of the present study was to investigate the prognostic significance of TUBB3 and topo-II expression levels in ATC. A total of 34 patients with ATC who received combination chemotherapy were enrolled in the present study. Immunohistochemical analysis was used to examine the expression of TUBB3, topo-II and $\mathrm{Ki}-67$ in tumor specimens obtained by surgical resection or biopsy. TUBB3 and topo-II were highly expressed in 38 and 53\% of the tumors, respectively. Progression-free survival (PFS) was significantly shorter in patients with high levels of TUBB3 compared with those with low levels of TUBB3 $(\mathrm{P}<0.01)$, whereas no significant difference in PFS between patients with high and low topo-II expression levels was observed $(\mathrm{P}=0.31)$. Patients with overexpression of TUBB3 or topo-II exhibited significantly shorter overall survival rates (OS) compared with those patients with low levels of expression of
\end{abstract}

Correspondence to: Dr Kyoichi Kaira, Department of Oncology Clinical Development, Gumna University Graduate School of Medicine, 3-39-15, Showa-machi, Meabashi, Gunma 371-8511, Japan E-mail: kkaira1970@yahoo.co.jp

Key words: thymic carcinoma, class III $\beta$-tubulin, topoisomerase-II, prognostic factor, immunohistochemistry these proteins (TUBB3; $\mathrm{P}=0.01$, topo-II; $\mathrm{P}=0.01$ ). Multivariate analysis demonstrated that a high level of TUBB3 expression was an independent unfavorable prognostic factor for OS, and a high level of topo-II expression tended to correlate with poor prognosis without statistical significance. Additionally, a subset analysis demonstrated that the treatment with taxanes, but not topo-II inhibitors, tended to prolong OS in patients with TUBB3 overexpression and there was significant survival advantage of chemoradiotherapy over chemotherapy in patients with topo-II overexpression. It was revealed that an enhanced expression of TUBB3 or topo-II was clearly associated with clinical outcomes in patients with ATC who received combination chemotherapy, including taxanes or topo-II inhibitors, suggesting the prognostic significance of these markers.

\section{Introduction}

Thymic carcinoma (TC) is a rare mediastinal malignancy with an annual incidence of 0.13 cases/100,000 population (1) and accounts for $\sim 5 \%$ of all thymic epithelial tumors (TETs) (2). TC has a propensity to invade the surrounding tissues and metastasize, and $\sim 2 / 3$ of all patients with TC are diagnosed with locally advanced or systemic disease (3). These aggressive features result in poor prognoses in the inoperable patients within Japan, with the 5-year survival rate of 24\% (4).

Although systemic chemotherapy is considered the standard of care for patients with advanced thymic carcinoma (ATC), an optimal regimen has not yet been established due to the disease rarity. Based on the results of a few small phase II trials and retrospective studies, combination chemotherapy, such as carboplatin/paclitaxel $(3,5)$, cisplatin/etoposide (6) and doxorubicin/cisplatin/vincristine/cyclophosphamide (ADOC) $(7,8)$, is a treatment option for patients with ATC in clinical practice. 
These regimens yield modest efficacy, however the response to chemotherapy and outcome vary considerably between patients. Therefore, biomarkers are required that predict the efficacy of chemotherapy and prognosis in patients with ATC receiving combination chemotherapy.

Taxane is a microtubule-stabilizing agent used in the treatment of several malignant tumors. Tubulin heterodimers consisting of $\alpha$ - and $\beta$-tubulin are the basic structural components of microtubules (9). Despite the existence of various $\alpha$ - and $\beta$-tubulin isotypes, several studies in different types of tumor have highlighted the association of class III $\beta$-tubulin (TUBB3) expression with resistance to taxane chemotherapy and poor prognosis (10-22). However, it remains unknown whether TUBB3 expression correlates with clinical outcome in patients with ATC.

Etoposide and anthracycline function by targeting topoisomerase II (topo-II), which serves an essential role during mitosis by generating transient DNA double-strand breaks and changing DNA topology and by controlling decatenation checkpoints and regulating sister chromosome segregation (23). Although previous studies have demonstrated the implication of topo-II expression in chemoresistance and poor prognoses in several malignancies (24-28), the clinical significance of topo-II expression in patients with ATC remains unknown. Therefore, immunohistochemical analysis of TUBB3 and topo-II expression was performed to elucidate whether the level of expression of these markers correlates with chemoresistance and clinical outcomes in patients with ATC.

\section{Materials and methods}

Patients and clinical outcome. A total of 40 patients with advanced or recurrent $\mathrm{TC}$ receiving combination chemotherapy at three Japanese institutions (Gunma Prefectural Cancer Center, Gunma University Hospital and National Hospital Organization Nishigunma Hospital, Gunma, Japan) were enrolled between April 1998 and April 2014. There were six patients excluded from the analysis as patient information or tumor specimen was not available for three, and the other three patients did not receive combination chemotherapy. As a result, 34 patients were eligible for the final analysis.

Baseline patient characteristics, data on antitumor effect of chemotherapy and survival data were retrospectively collected from the medical records of the enrolled patients. The clinical stage of each TC case was determined according to the Masaoka-Koga classification (29). The histological type was assessed according to the 2004 World Health Organization histological classification (30). Progression-free survival (PFS) was calculated from the beginning of the treatment for advanced or recurrent disease to the date of disease progression or mortality due to any cause. Similarly, overall survival (OS) was calculated until the date of mortality or the last follow-up consultation. The protocol was approved by the institutional review board of each institution (Gunma Prefectural Cancer Center, Gunma University Hospital and National Hospital Organization Nishigunma Hospital) and complied with the Declaration of Helsinki.

Immunohistochemical staining. Tumor specimens were obtained by surgical excision or biopsy. Immunohistochemical staining procedure has been previously described $(31,32)$. Antibodies used in the present study were as follows: TUBB3 mouse monoclonal antibody (cat. no., MMS-435P; dilution, 1:100; Covance, Inc., Princeton, NJ, USA), topo-II rabbit polyclonal antibody (cat. no., ab180393; dilution, 1:100; Abcam, Tokyo, Japan), and MIB-1 mouse monoclonal antibody, specific for human nuclear antigen Ki-67 (cat. no., M7240; dilution 1:40; Dako; Agilent Technologies, Inc., Santa Clara, CA, USA).

Cells were considered positive for TUBB3/topo-II/Ki-67 if the staining was present in the cytoplasm or the nuclei. The proportion of TUBB3/topo-II-positive cells was assessed by a semi-quantitative scoring method where samples were assigned a score based on the percentage of positive cells: $1, \leq 10 \% ; 2,>10-\leq 25 \% ; 3,>25-\leq 50 \% ; 4,>50-\leq 75 \% ; 5,>75 \%$. Samples with scores of 1 and 2 were considered to exhibit low levels of expression, whereas those with scores of 3,4 and 5 were considered to exhibit high levels of expression. Expression of Ki-67 was evaluated using Ki-67 labeling index (KI), which was defined as the proportion of positive cells among 1,000 tumor cells in each sample. As the samples used in the analysis also included biopsy specimens, only the presence of the staining, but not the intensity, was considered. Sections were examined by light microscopy in a blinded fashion by at least two investigators. In case of discrepancies, the two investigators simultaneously evaluated the slides until a consensus was reached. The investigators were blinded to patient outcomes.

Statistical analysis. $\mathrm{P}<0.05$ was considered to indicate a statistically significant difference. The association between immunohistochemical staining and patient characteristics was examined using Fisher's exact test. The correlation between different variables was analysed using the nonparametric Spearman's rank test. The Kaplan-Meier method was used to estimate survival, and the survival differences were analysed by the log-rank test. Multivariate analyses were performed using the Cox proportional hazards model to identify independent prognostic factors. Statistical analysis was performed using GraphPad Prism 6 software (Graph Pad Software, Inc., La Jolla, CA, USA) and EZR (Saitama Medical Center, Jichi Medical University, Saitama, Japan) for Windows.

\section{Results}

Patient characteristics. Patient characteristics are summarized in Table I. The study included 21 males (68\%) and 13 females (32\%) with a median age of 62 years, range, 36-75 years. Prior to the enrollment, 10 patients (29\%) experienced recurrence subsequent to curative resection of the primary tumor. According to the Masaoka-Koga staging, 5 patients (15\%) were stage III, $11(32 \%)$ were stage IVa, and $18(53 \%)$ were stage IVb. The main histological types were squamous cell carcinoma (SqCC), 19 patients (56\%) undifferentiated carcinoma, 6 patients (18\%) and carcinoid tumor, 4 patients (12\%). A total of 21 patients (62\%) received chemotherapy alone and 13 (38\%) received chemoradiotherapy (CRT) as the initial treatment. The main first-line chemotherapy regimens included etoposide-based doublet, 14 patients (41\%) taxane-based doublet, 13 patients (38\%) and ADOC, 4 patients 
Table I. Baseline characteristics of 34 patients with advanced thymic carcinoma treated with chemotherapy.

\begin{tabular}{|c|c|}
\hline Characteristic & Number \\
\hline Age, median years (range) & $62(36-75)$ \\
\hline Gender, male/female & $21 / 13$ \\
\hline Smoking, yes/no & $17 / 17$ \\
\hline Post-operation recurrence & 10 \\
\hline $\begin{array}{l}\text { Stage (Masaoka-Koga } \\
\text { classification), III/IVa/IVb }\end{array}$ & $5 / 11 / 18$ \\
\hline $\begin{array}{l}\text { Long diameter of primary } \\
\text { tumor }^{\mathrm{a}} \text {, median (range) }\end{array}$ & $\begin{array}{c}70 \mathrm{~mm} \\
(26-120 \mathrm{~mm})\end{array}$ \\
\hline PS, $0 / 1 / 2$ & $22 / 11 / 1$ \\
\hline \multicolumn{2}{|l|}{ Metastatic site } \\
\hline Pleural dissemination & 14 \\
\hline Pericardial dissemination & 8 \\
\hline Lung & 12 \\
\hline Lymph node & 8 \\
\hline Bone & 3 \\
\hline Liver & 3 \\
\hline Others & 4 \\
\hline \multicolumn{2}{|l|}{ Histology } \\
\hline Squamous cell carcinoma & 19 \\
\hline Carcinoid tumor & 4 \\
\hline $\begin{array}{l}\text { Poorly differentiated } \\
\text { neuroendocrine carcinoma }\end{array}$ & 2 \\
\hline Undifferentiated carcinoma & 6 \\
\hline Others & 2 \\
\hline Unknown & 1 \\
\hline \multicolumn{2}{|l|}{ Initial treatment } \\
\hline Chemoradiotherapy & $\begin{array}{c}13 \\
(\text { seq. } 9, \text { conc. } 4)\end{array}$ \\
\hline Chemotherapy & 21 \\
\hline \multicolumn{2}{|l|}{ First-line chemotherapy regimen } \\
\hline $\mathrm{CBDCA}+\mathrm{PTX}$ & 11 \\
\hline $\mathrm{CDDP} / \mathrm{CBDCA}+\mathrm{DOC}$ & 2 \\
\hline CDDP/CBDCA+ETP & 14 \\
\hline ADOC & 4 \\
\hline Others & 3 \\
\hline \multicolumn{2}{|l|}{ Post-progression therapy } \\
\hline Single agent chemotherapy & 7 \\
\hline Combination chemotherapy & 12 \\
\hline Surgery & 2 \\
\hline Palliative radiation & 12 \\
\hline Others & 2 \\
\hline Best supportive care alone & 6 \\
\hline
\end{tabular}

PS, performance status; seq, sequential; conc, concurrent; CBDCA, carboplatin; PTX, paclitaxel; CDDP, cisplatin; DOC, docetaxel; ETP, etoposide; ADOC, doxorubicin+cisplatin+vincristine+cyclophospha mide. ${ }^{a}$ Data on long diameter of primary tumor was not available for five patients.
(12\%). Subsequent to the failure of initial treatment, the two main subsequent therapies were chemotherapy, 19 patients $(56 \%)$ and palliative radiation, 12 patients $(35 \%)$.

Immunohistochemical analysis. Immunohistochemical analysis was performed on specimens from 24 primary lesions and 10 metastatic lesions. The expression levels of TUBB3, topo-II and Ki-67 were evaluable in 32, 34 and 34 patients, respectively. Fig. 1 demonstrates representative images of TUBB3 and topo-II staining. KI ranged from 0 to $68 \%$ with the median value of $20 \%$, and the cutoff value was set at $20 \%$. High expression levels of TUBB3, topo-II and Ki-67 were detected in $20(62 \%), 18(53 \%)$ and 17 tumors (50\%), respectively. The mean scores of TUBB3 and topo-II were $2.09 \pm 1.28$ and $2.59 \pm 0.99$, respectively.

Patient characteristics according to the expression level of TUBB3/topo-II. Detailed data on patient characteristics according to the expression level of TUBB3 and topo-II are summarized in Table II. Although TUBB3 expression and any clinicopathological factors were not significantly associated, topo-II expression significantly correlated with age and $\mathrm{KI}$ $(\mathrm{P}<0.01$ and $\mathrm{P}=0.02$, respectively). Additionally, Spearman's rank test demonstrated that topo-II expression positively correlated with age $(\mathrm{r}=0.57, \mathrm{P}<0.01)$ and $\mathrm{KI}(\mathrm{r}=0.42, \mathrm{P}=0.02)$.

Response to first-line chemotherapy. Overall response rate (ORR) in all 34 patients was $35 \%$. ORRs in patients with high and low TUBB3 expression were $36 \%$ ( 5 in 14 patients) and $35 \%$ (7 in 20 patients), respectively $(\mathrm{P}=1.00)$. In patients with high and low topo-II expression, ORRs were 39\% (7 in 18 patients) and $31 \%$ (5 in 16 patients), respectively $(\mathrm{P}=0.73)$. Amongst patients treated with taxanes, ORRs were $25 \%$ ( 1 in 4 patients) and $29 \%$ ( 2 in 7 patients) in patients with high and low TUBB3 expression, respectively $(\mathrm{P}=1.00)$. Finally, amongst patients treated with topo-II inhibitors, ORRs in patients with high and low topo-II expression levels were $56 \%$ (5 in 9 patients) and $33 \%$ (4 in 12 patients), respectively $(\mathrm{P}=0.40)$.

Survival analysis and clinicopathological factors. During the median follow-up period of 27.5 months, range, 1.3-119.7 months, 33 patients experienced disease progression and there were 23 patient mortalities. The median PFS was 7.4 and the median OS was 37.1 months. The two-five-year OS rates were 62.5 and $25.3 \%$, respectively. Patients with high TUBB3 expression exhibited a shorter median PFS compared with patients with low TUBB3 expression, 6.4 vs. 10.5 months, respectively $(\mathrm{P}<0.01$; Fig. $2 \mathrm{~A})$, whilst no significant difference was observed in the length of median PFS was observed between patients with high and low topo-II expression, 6.6 vs. 7.7 months, respectively ( $\mathrm{P}=0.31$; Fig. $2 \mathrm{~B})$. Similarly, patients with high TUBB3 expression exhibited shorter median OS compared with patients with low TUBB3 expression, 14.4 vs. 52.3 months, respectively ( $\mathrm{P}=0.01$; Fig. $2 \mathrm{C}$ ), and the median OS in patients with high and low topo-ii expression was 23.9 and 58.9 months, respectively ( $\mathrm{P}=0.01$; Fig. 2D). Amongst patients with initial treatment consisting of chemotherapy alone, subjects with TUBB3 overexpression tended to achieve shorter OS compared with those with TUBB3 low expression, although this difference did not appear statistically significant 

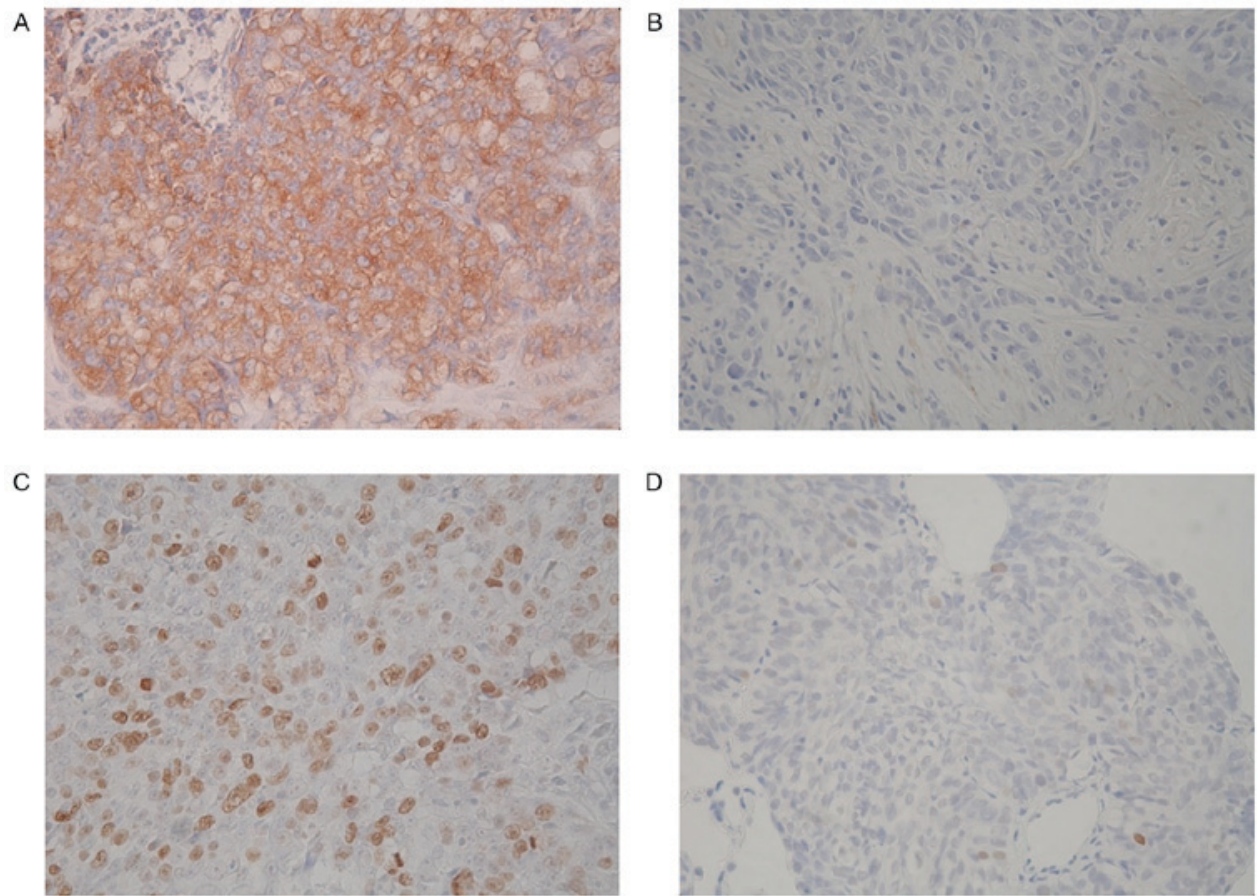

Figure 1. Immunohistochemical staining of topoisomerase-II and TUBB3 in thymic carcinoma at magnification, $x 400$. Immunoshistochemical staining was performed by diamino benzidine method and was evaluated using an optical light microscope. (A) Strong TUBB3 staining is present in cytoplasm, score 5 . (B) TUBB3 staining is barely present, score 1. (C) Topo-II is strongly stained in the nuclei, score 4. (D) Topo-II staining is barely visible, score 1. TUBB3, class III $\beta$-tubulin.

$(\mathrm{P}=0.051)$, whereas high topo-II expression significantly correlated with poor $\mathrm{OS}(\mathrm{P}=0.02)$.

Univariate analysis demonstrated that older age, histological type, SqCC vs. others, and high levels of TUBB3 and topo-II expression were significant prognostic factors associated with decreased OS (Table III). Multivariate analysis demonstrated that age $(\mathrm{HR}, 2.95 ; \mathrm{P}=0.04)$ and TUBB3 expression (HR, 3.05; $\mathrm{P}=0.04$ ) were independent factors for predicting OS (Table III). High topo-II expression tended to correlate with poor prognosis without statistical significance (HR, 2.13; $\mathrm{P}=0.17$; Table III).

Survival analysis according to treatment and TUBB3/topo-II expression. A survival analysis was conducted in patients with TUBB3 overexpression who received taxanes $(n=12)$ or topo-II inhibitors $(\mathrm{n}=12)$ at any time during the treatment. Taxane treatment demonstrated a tendency to prolong OS $(\mathrm{P}=0.08$; Fig. 3A), whereas treatment with topo-II inhibitors tended to shorten $\mathrm{OS}$ ( $\mathrm{P}=0.15$; Fig. 3B).

A survival analysis was also performed in patients initially treated with CRT $(n=13)$ or chemotherapy alone $(n=21)$. A trend toward prolonged OS was observed in all patients treated with CRT in comparison with those treated with chemotherapy alone ( $\mathrm{P}=0.05 ;$ Fig. $4 \mathrm{~A})$. OS did not differ significantly between patients treated with CRT or chemotherapy alone with regard to TUBB3 status (low TUBB3, $\mathrm{P}=0.19$; high TUBB3, $\mathrm{P}=0.37$; Fig. 4B and $\mathrm{C}$, respectively). In contrast, the survival benefit of CRT over chemotherapy alone was statistically significant in patients with high topo-II expression ( $\mathrm{P}=0.01$; Fig. $4 \mathrm{E})$, whereas in patients with low levels of topo-II, OS was equivalent in the two treatment groups $(\mathrm{P}=0.32$; Fig. 4D).

\section{Discussion}

In the present study, it was revealed that a high level of TUBB3 expression was a significant predictive marker for shorter PFS and OS, and a high level of topo-ii expression was also correlated with poor OS in patients with ATC receiving combination chemotherapy including taxanes or topo-II inhibitors. Additionally, it was noted that treatment with taxanes, but not topo-II inhibitors, tended to prolong OS in patients with TUBB3 overexpression, and a survival advantage of CRT compared with chemotherapy was detected in patients with topo-II overexpression. To the best of our knowledge, this is the first study in patients with ATC to demonstrate the prognostic significance of TUBB3 and topo-II expression.

A significant association between TUBB3 expression level and response to taxane-based regimen has been observed in patients with NSCLC (10-13) and gastric cancer $(21,22)$. Similar results have been demonstrated in patients with TET receiving carboplatin/paclitaxel chemotherapy (33). These results indicated that TUBB3 expression may be a potential candidate for a predictive marker of response to taxane-based chemotherapy. Conversely, the significance of TUBB3 expression for predicting response to chemotherapy was not confirmed. Results consistent with those of the present study have been demonstrated in patients with unresectable ovarian cancer (14) and advanced NSCLC $(15,16)$. Therefore, whether TUBB3 expression predicts effectiveness of taxanes remains debatable. Previous preclinical studies have indicated that TUBB3 promotes tumorigenesis, metastases, and anoikis resistance (34-36). Immunohistochemical analysis of TETs has suggested that TUBB3 overexpression correlates with tumor aggressiveness, regulation of cell cycle and angiogenesis (33). 
Table II. Patient characteristics according to biomarkers.

\begin{tabular}{|c|c|c|c|c|c|c|}
\hline \multirow[b]{2}{*}{ Parameter } & \multicolumn{3}{|c|}{ TUBB $^{\mathrm{b}}$} & \multicolumn{3}{|c|}{ Topo-II } \\
\hline & High $(n=12)$ & Low $(n=20)$ & $\mathrm{P}$-value & High $(n=18)$ & Low $(n=16)$ & $\mathrm{P}$-value \\
\hline \multicolumn{7}{|l|}{ Age, years } \\
\hline$\leq 61$ & 7 & 10 & 0.73 & 5 & 13 & $<0.01^{\mathrm{c}}$ \\
\hline$\geq 62$ & 5 & 10 & & 13 & 3 & \\
\hline \multicolumn{7}{|l|}{ Gender } \\
\hline Male & 6 & 14 & 0.29 & 13 & 8 & 0.29 \\
\hline Female & 6 & 6 & & 5 & 8 & \\
\hline \multicolumn{7}{|l|}{ Smoking status } \\
\hline Smoker & 7 & 10 & 0.73 & 10 & 7 & 0.73 \\
\hline Non-smoker & 5 & 10 & & 8 & 9 & \\
\hline \multicolumn{7}{|c|}{ Stage (Masaoka-Koga) } \\
\hline III & 0 & 5 & 0.13 & 2 & 3 & 0.65 \\
\hline IV & 12 & 15 & & 16 & 13 & \\
\hline \multicolumn{7}{|l|}{ Histology } \\
\hline Squamous & 4 & 14 & 0.07 & 11 & 8 & 0.73 \\
\hline Non-squamous & 8 & 6 & & 7 & 8 & \\
\hline \multicolumn{7}{|l|}{ Tumor size } \\
\hline$<70 \mathrm{~mm}$ & 7 & 11 & 1.00 & 10 & 9 & 1.00 \\
\hline$\geq 70 \mathrm{~mm}$ & 5 & 9 & & 8 & 7 & \\
\hline \multicolumn{7}{|l|}{ PS } \\
\hline 0 & 7 & 13 & 0.72 & 13 & 9 & 0.48 \\
\hline $1 / 2$ & 5 & 7 & & 5 & 7 & \\
\hline \multicolumn{7}{|l|}{ Initial treatment } \\
\hline CRT & 3 & 9 & 0.45 & 7 & 6 & 1.00 \\
\hline Chemotherapy & 9 & 11 & & 11 & 10 & \\
\hline \multicolumn{7}{|c|}{ Post-progression chemotherapy } \\
\hline Yes & 7 & 11 & 1.00 & 12 & 7 & 0.30 \\
\hline No & 5 & 9 & & 6 & 9 & \\
\hline \multicolumn{7}{|c|}{ Ki-67 labeling index } \\
\hline$<20$ & 6 & 10 & 1.00 & 5 & 12 & $0.02^{c}$ \\
\hline$\geq 20$ & 6 & 10 & & 13 & 4 & \\
\hline \multicolumn{7}{|l|}{ TUBB3 $^{\mathrm{a}}$} \\
\hline High & - & - & - & 9 & 3 & 0.08 \\
\hline Low & - & - & & 8 & 12 & \\
\hline \multicolumn{7}{|l|}{ Topo-II } \\
\hline High & 9 & 8 & 0.08 & - & - & - \\
\hline Low & 3 & 12 & & - & - & \\
\hline
\end{tabular}

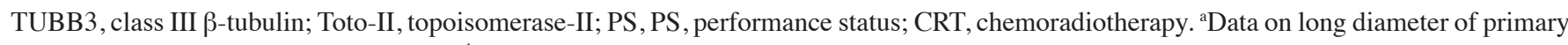
tumor was not available for five patients; ${ }^{b}$ Data on TUBB3 status was not available for two patients. ${ }^{\mathrm{C}} \mathrm{P}<0.05$. The expression levels of Topo-II were significantly correlated with age and Ki-67 labeling index.

Thus, TUBB3 may contribute to the intrinsic aggressiveness of ATC as opposed to the resistance of ATC to chemotherapy. Validation of the prognostic significance of TUBB3 overexpression and additional investigations of biological mechanisms underlying clinical effects of TUBB3 overexpression are required.
The guidelines recommend less toxic carboplatin/paclitaxel chemotherapy compared with anthracycline-based regimens (37). However, conventional anthracycline-based regimens remain frequently administered as they appear more effective compared with carboplatin/paclitaxel combinations $(3,5,7,8)$. Notably, the data of the present study 
A

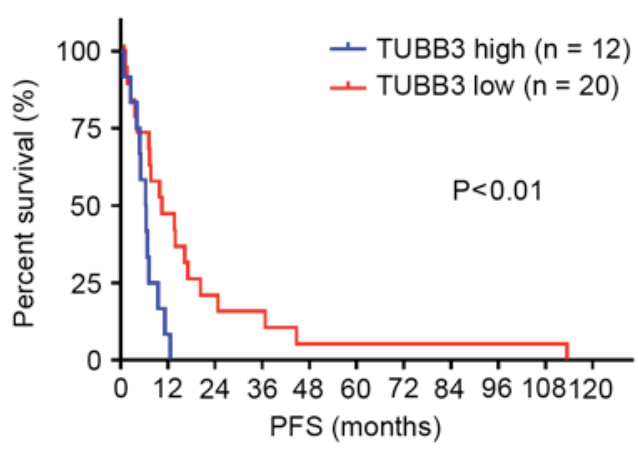

C

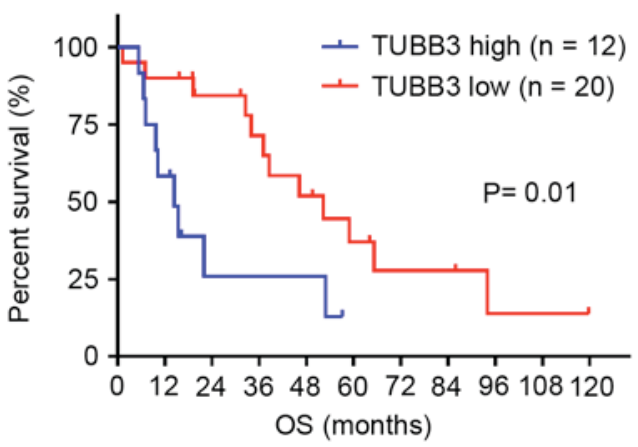

B

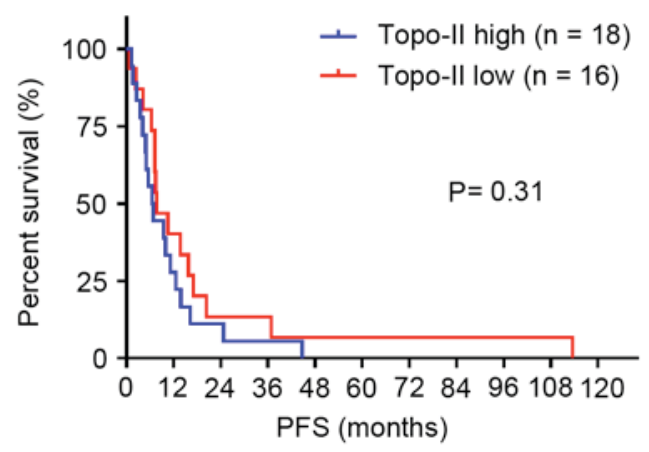

D

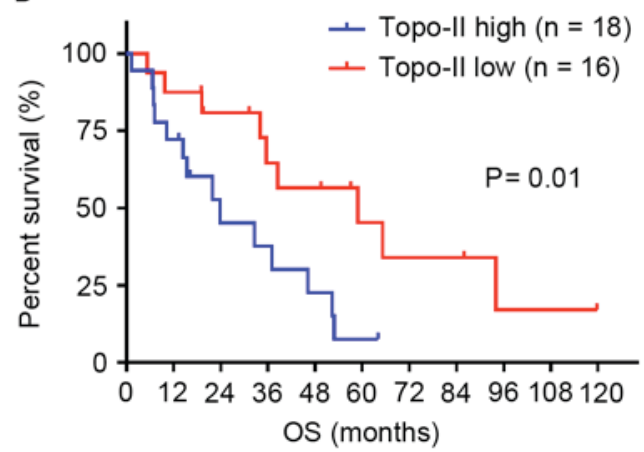

Figure 2. Survival analysis using the Kaplan-Meier method. (A) The median PFS for patients with high TUBB3 expression was significantly shorter compared with patients with low TUBB3 expression, median, 6.4 vs. 10.5 months, respectively (HR, 2.44; $\mathrm{P}<0.01$ ). (B) No significant difference was observed in median PFS between patients with high and low topo-II expression, median, 6.6 vs. 7.7 months, respectively (HR, 1.42; $\mathrm{P}=0.31)$. (C) Median OS for patients with high TUBB3 expression was significantly shorter compared with patients with low TUBB3 expression, median, 14.4 vs. 52.3 months, respectively (HR, 2.73; $\mathrm{P}=0.01$ ). (D) Median OS for patients with high topo-II expression was significantly shorter compared with patients with low topo-II expression, median, 23.9 vs. 58.9 months, respectively (HR, 2.62; P=0.01). Topo-II, topoisomerase-II; TUBB3, class III $\beta$-tubulin, PFS, progression-free survival; OS, overall survival rate.

suggest that taxanes, but not topo-II inhibitors, may improve OS in patients with high TUBB3 expression. Additionally, Galmarini et al (38) have demonstrated that patients with advanced breast cancer with high TUBB3 expression exhibited an improved response to docetaxel compared with to doxorubicin. Thus, TUBB3 expression may be a useful marker that provides an indication for taxane-based regimen in patients with ATC. However, as a direct correlation between the type of treatment and tumor response was not observed, these results must be interpreted with caution.

Previous in vitro studies $(39,40)$ and clinical retrospective studies (41-43) have suggested that an increased level of topo-II may predict a better response to topo-II inhibitors. In addition, Liu et al (44) have demonstrated that topo-II overexpression was significantly correlated with chemosensitivity in 20 patients with TET receiving anthracycline-based chemotherapy. In contrast, the data of the present study did not confirm the significance of topo-II overexpression in predicting responses to chemotherapy. This discrepancy may be attributed to the fact that, contrary to the study by Liu et al (44), the present study was restricted to patients with ATC and included various chemotherapy regimens. In agreement with the data of the present study, no obvious association between topo-II expression and response to chemotherapy was detected in lung cancer $(24-26,45)$. Instead of predicting chemosensitivity, high levels of topo-II expression may serve as a specific marker of cell proliferation, invasive behavior and unfavorable prognosis. Topo-II has been revealed to be involved in cell proliferation and tumorigenicity in prostate cancer cells (28). Clinical retrospective studies have demonstrated that elevated levels of topo-II were correlated with increased levels of Ki-67 and metastasis $(42,46)$ and poor clinical outcomes (24-28) in various types of malignancy. These data support the results of the present study that topo-II overexpression was significantly associated with Ki-67 overexpression and unfavorable prognosis. In accordance with the data of a large-scale randomized trial in breast cancer (27), the present study also demonstrated a significant correlation between topo-II overexpression and old age. This association is consistent with the results of the previous preclinical studies suggesting that topo-II may interact with several DNA metabolism proteins associated with human aging (47). Notably, the present study demonstrated that old age was also an independent prognostic factor for shorter OS. These data indicate that topo-II may confer the propensity to exhibit poor prognoses through a mechanism involved in aging. However, as the present study did not confirm this conjecture and did not exclude the possibility that topo-II overexpression was only a confounding factor, the prognostic value of topo-II and the association of topo-ii with $\mathrm{Ki}-67$ and age requires additional investigation in other studies.

The clinical benefit of radiation in ATC has not been evaluated in prospective trials, and the clinicopathological backgrounds in which radiation confers higher effectiveness 
Table III. Cox regression analysis of OS.

A, Univariate analysis.

\begin{tabular}{|c|c|c|c|}
\hline Variables & HR & $95 \% \mathrm{CI}$ & P-value \\
\hline Age ( $\geq 62$ vs. $\leq 61$, years $)$ & 3.28 & $1.79-10.3$ & $<0.01^{\mathrm{a}}$ \\
\hline Gender (male vs. female) & 0.92 & $0.40-2.15$ & 0.85 \\
\hline Smoking (yes vs. no) & 0.78 & $0.34-1.77$ & 0.55 \\
\hline Stage (III vs. IV) & 0.87 & $0.31-2.43$ & 0.79 \\
\hline PS (0 vs. 1/2) & 1.13 & $0.50-2.63$ & 0.76 \\
\hline Long diameter of primary tumor ( $<70 \mathrm{vs} . \geq 70 \mathrm{~mm}$ ) & 1.03 & $0.43-2.44$ & 0.95 \\
\hline Initial treatment (CRT vs. chemotherapy) & 0.42 & $0.18-1.01$ & 0.05 \\
\hline Histology (Sq. vs. others) & 0.38 & $0.14-0.80$ & $0.02^{\mathrm{a}}$ \\
\hline Ki-67 labeling index ( $\geq 15$ vs. $\leq 15)$ & 1.05 & $0.47-2.39$ & 0.90 \\
\hline TUBB3 (high vs. low) & 2.73 & $1.40-11.2$ & $0.01^{\mathrm{a}}$ \\
\hline Topo-II (high vs. low) & 2.62 & $1.31-7.09$ & $0.01^{\mathrm{a}}$ \\
\hline
\end{tabular}

B, Multivariate analysis.

\begin{tabular}{|c|c|c|c|}
\hline Variables & HR & $95 \% \mathrm{CI}$ & P-value \\
\hline Age ( $\geq 62$ vs. $\leq 61$, years $)$ & 2.95 & $1.06-8.21$ & $0.04^{\mathrm{a}}$ \\
\hline TUBB3 (high vs. low) & 3.05 & $1.04-8.90$ & $0.04^{\mathrm{a}}$ \\
\hline Topo-II (high vs. low) & 2.13 & $0.72-6.28$ & 0.17 \\
\hline
\end{tabular}

PS, performance status; CRT, chemoradiotherapy; Sq, squamous cell carcinoma. ${ }^{a} \mathrm{P}<0.05$. While univariate analysis demonstrated that $\mathrm{OS}$ was significantly associated with age, histology, TUBB3 expression and Topo-II expression, multivariate analysis demonstrated that OS was significantly correlated with age and TUBB3 expression.

A

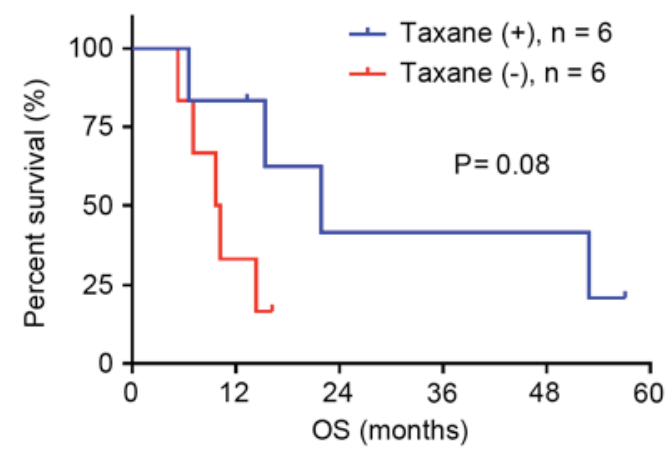

B

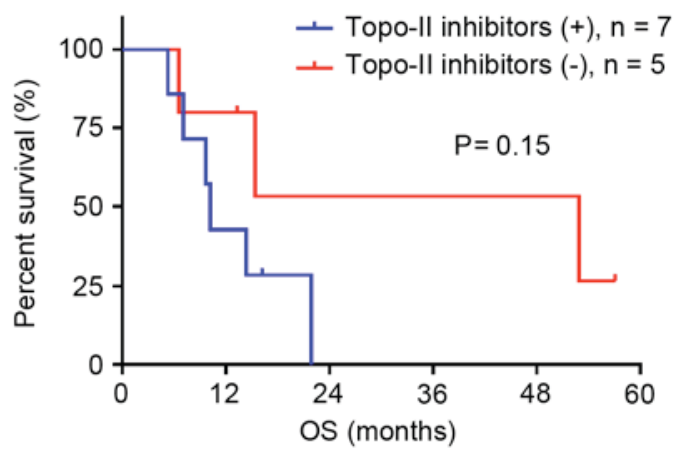

Figure 3. Kaplan-Meier curves demonstrate OS in patients with high TUBB3 expression (n=12) according to administration of (A) taxanes and (B) topo-II inhibitors such as etoposide, doxorubicin and amrubicin. Although the difference in OS between the two groups is not statistically significant, patients treated with taxanes tend to have a longer survival compared with patients treated with topo-II inhibitors. Topo-II, topoisomerase-II; OS, overall survival rate.

remain unclear. The present study indicated that in patients with ATC, topo-II overexpression may serve as a predictive marker for a survival advantage of CRT compared with chemotherapy alone. In agreement with this result, several preclinical studies have revealed that elevated topo-II expression levels positively correlate with radiation-induced chromatid breaks and increased radiosensitivity in various types of cancer cell lines (48-50). However, additional studies are required to elucidate the role of topo-II in augmentation of radiosensitivity in ATC.
The present study has several limitations. Firstly, it is retrospective and possesses a small sample size. However, notably, the present study is the largest assessing biomarkers for ATC as such evaluation is difficult in large-scale prospective studies. Secondly, biopsy specimens, whenever sufficient and adequate for evaluation, were also included in immunohistochemical examination. Finally, the inclusion of patients who received CRT as initial treatment may confound the interpretation of the results. Therefore, the data from patients initially treated with chemotherapy alone 
A.

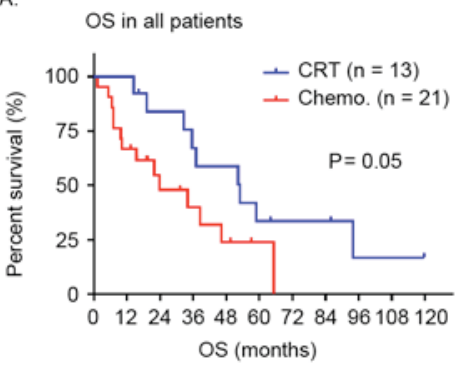

D.

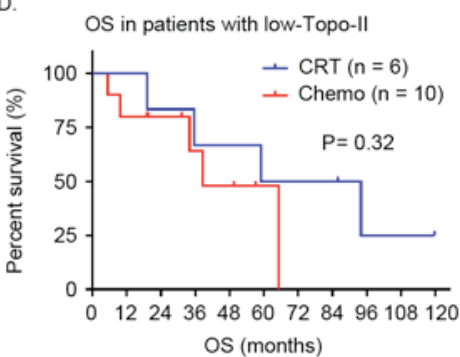

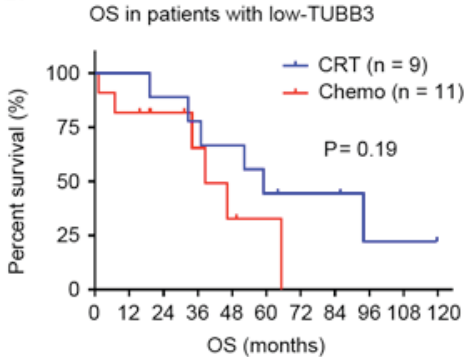

$\mathrm{E}$

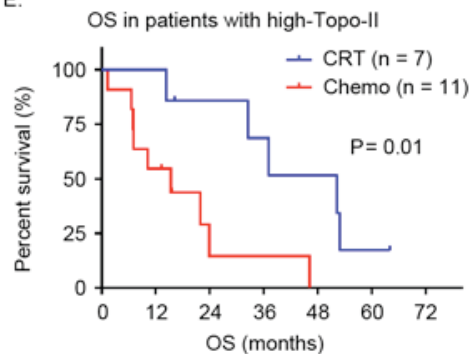

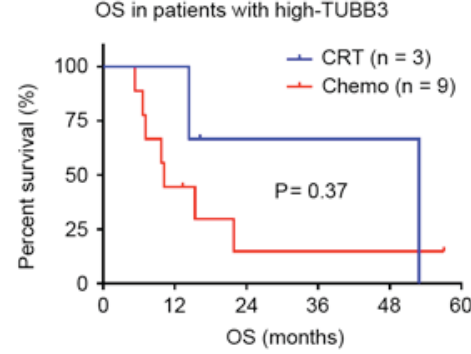

Figure 4. Kaplan-Meier curves demonstrate OS according to initial treatment. (A) OS in patients treated with CRT tended to be longer compared with patients treated with chemotherapy alone, median OS, 52.9 vs. 23.9 months, respectively $(\mathrm{HR}, 0.42 ; \mathrm{P}=0.05)$. (B and C) There was no significant difference in OS according to initial treatment between patients with low TUBB3 expression, median OS 58.9 vs. 38.5 months, respectively (HR, 0.42; P=0.19) and high TUBB3 expression, median OS, 52.9 vs. 10.2 months, respectively (HR, 0.53; P=0.37). (D and E) Among patients with high topo-II expression, OS in patients treated with CRT was significantly longer compared with patients treated with chemotherapy alone, median OS, 52.3 vs. 15.4 months, respectively (HR, 0.22 ; $\mathrm{P}=0.01$ ), whereas no significant difference was observed in OS according to initial treatment among patients with low topo-II expression median OS, 76.4 vs. 39.8 months (HR, 0.48; $\mathrm{P}=0.32$ ). Topo-II, topoisomerase-II; TUBB3, class III $\beta$-tubulin; OS, overall survival rate.

were separately analyzed. Results consistent with previous studies were obtained, suggesting that the overexpression of TUBB3/topo-II serves an important role in predicting prognoses regardless of the type of initial treatment. These data propose the prospect of personalized therapy according to molecular markers, such as TUBB3 and topo-II in ATC. In addition, the present study hypothesizes that the identification of TUBB3 and topo-II as the novel prognostic factors may assist in the development of therapeutic agents and treatment strategies for patients with ATC possessing unfavorable prognosis.

In conclusion, a significant correlation between overexpression of TUBB3/topo-II and poor clinical outcomes in patients with ATC receiving combination chemotherapy including taxanes or topo-ii inhibitors was demonstrated. Additionally, these markers may be helpful in determining the optimal chemotherapy regimen and in selecting patients with the indication for CRT. Additional validation studies are required to verify the clinical effect of these markers.

\section{Acknowledgements}

The authors would like to thank Dr Akira Mogi, Department of General Surgical Science, Dr Kimihiro Shimizu, Department of Thoracic Visceral Organ Surgery and Dr Masataka Maeno, Department of Medicine and Biological Science, for data collection. They would also like to express gratitude to all the staff in the pathology departments of the Gunma University Hospital, Gunma Prefectural Cancer Center and National Hospital Organization Nishigunma Hospital for technical assistance in immunohistochemical analysis. This work was supported in part by grant no. 26461154 (awarded to Dr Kyoichi Kaira) from the Ministry of Education, Culture, Sports, Science and Technology, Japan.

\section{References}

1. Kelly RJ, Petrini I, Rajan A, Wang Y and Giaccone G: Thymic malignancies: From clinical management to targeted therapies. J Clin Oncol 29: 4820-4827, 2011.

2. Engels EA and Pfeiffer RM: Malignant thymoma in the United States: Demographic patterns in incidence and associations with subsequent malignancies. Int J Cancer 105: 546-551, 2003.

3. Lemma GL, Lee JW, Aisner SC, Langer CJ, Tester WJ, Johnson DH and Loehrer PJ Sr: Phase II study of carboplatin and paclitaxel in advanced thymoma and thymic carcinoma. J Clin Oncol 29: 2060-2065, 2011.

4. Kondo K and Monden Y: Therapy for thymic epithelial tumors: A clinical study of 1,320 patients from Japan. Ann Thorac Surg 76: 878-885, 2003.

5. Hirai F, Yamanaka T, Taguchi K, Daga H, Ono A, Tanaka K, Kogure Y, Shimizu J, Kimura T, Fukuoka J, et al: A multicenter phase II study of carboplatin and paclitaxel for advanced thymic carcinoma: WJOG4207L. Ann Oncol 26: 363-368, 2015.

6. Giaccone G, Ardizzoni A, Kirkpatrick A, Clerico M, Sahmoud T and van Zandwijk N: Cisplatin and etoposide combination chemotherapy for locally advanced or metastatic thymoma. A phase II study of the European organization for research and treatment of cancer lung cancer cooperative group. J Clin Oncol 14: 814-820, 1996.

7. Fornasiero A, Daniele O, Ghiotto C, Piazza M, Fiore-Donati L, Calabró F, Rea F and Fiorentino MV: Chemotherapy for invasive thymoma. A 13-year experience. Cancer 68: 30-33, 1991.

8. Agatsuma T, Koizumi T, Kanda S, Ito M, Urushihata K, Yamamoto H, Hanaoka M and Kubo K: Combination chemotherapy with doxorubicin, vincristine, cyclophosphamide, and platinum compounds for advanced thymic carcinoma. J Thorac Oncol 6: 2130-2134, 2011.

9. Kavallaris M: Microtubules and resistance to tubulin-binding agents. Nat Rev Cancer 10: 194-204, 2010. 
10. Levallet G, Bergot E, Antoine M, Creveuil C, Santos AO, Beau-Faller M, de Fraipont F, Brambilla E, Levallet J, Morin F, et al: High TUBB3 expression, an independent prognostic marker in patients with early non-small cell lung cancer treated by preoperative chemotherapy, is regulated by K-Ras signaling pathway. Mol Cancer Ther 11: 1203-1213, 2012.

11. Huang CL, Kadota K, Liu D, Ueno M, Nakasima N, Ishikawa S, Gotoh M, Misaki N, Chang SS and Yokomise H: Expression of ERCC1 and class III $\beta$-tubulin is associated with the survival of resected stage III non-small cell lung cancer patients treated with induction chemoradiotherapy using carboplatin-taxane. Exp Ther Med 1: 445-451, 2010

12. Sève $P$ and Dumontet C: Is class III beta-tubulin a predictive factor in patients receiving tubulin-binding agents? Lancet Oncol 9: 168-175, 2008

13. Kaira K, Takahashi T, Murakami H, Shukuya T, Kenmotsu H, Ono A, Naito T, Tsuya A, Nakamura Y, Endo M, et al: The role of $\beta$ III-tubulin in non-small cell lung cancer patients treated by taxane-based chemotherapy. Int J Clin Oncol 18: 371-379, 2013.

14. Ferrandina G, Zannoni GF, Martinelli E, Paglia A, Gallotta V, Mozzetti S, Scambia G and Ferlini C: Class III beta-tubulin overexpression is a marker of poor clinical outcome in advanced ovarian cancer patients. Clin Cancer Res 12: 2774-2779, 2006.

15. Vilmar AC, Santoni-Rugiu E and Sørensen JB: Class III $\beta$-tubulin in advanced NSCLC of adenocarcinoma subtype predicts superior outcome in a randomized trial. Clin Cancer Res 17: 5205-5214, 2011.

16. Edelman MJ, Schneider CP, Tsai CM, Kim HT, Quoix E, Luft AV, Kaleta R, Mukhopadhyay P, Trifan OC, Whitaker L and Reck M: Randomized phase II study of ixabepilone or paclitaxel plus carboplatin in patients with non-small-cell lung cancer prospectively stratified by beta-3 tubulin status. J Clin Oncol 31: 1990-1996, 2013.

17. Roque DM, Buza N, Glasgow M, Bellone S, Bortolomai I, Gasparrini S, Cocco E, Ratner E, Silasi DA, Azodi M, et al: Class III $\beta$-tubulin overexpression within the tumor microenvironment is a prognostic biomarker for poor overall survival in ovarian cancer patients treated with neoadjuvant carboplatin/paclitaxel. Clin Exp Metastasis 31: 101-110, 2014.

18. Roque DM,Bellone S, English DP, Buza N, Cocco E, Gasparrini S, Bortolomai I, Ratner E, Silasi DA, Azodi M, et al: Tubulin- $\beta$-III overexpression by uterine serous carcinomas is a marker for poor overall survival after platinum/taxane chemotherapy and sensitivity to epothilones. Cancer 119: 2582-2592, 2013

19. Hasegawa S, Miyoshi Y, Egawa C, Ishitobi M, Taguchi T, Tamaki Y, Monden M and Noguchi S: Prediction of response to docetaxel by quantitative analysis of class I and III beta-tubulin isotype mRNA expression in human breast cancers. Clin Cancer Res 9: 2992-2997, 2003

20. Paradiso A, Mangia A, Chiriatti A, Tommasi S, Zito A, Latorre A, Schittulli F and Lorusso V: Biomarkers predictive for clinical efficacy of taxol-based chemotherapy in advanced breast cancer. Ann Oncol 16 (Suppl 4): iv14-iv19, 2005.

21. Hwang JE, Hong JY, Kim K, Kim SH, Choi WY, Kim MJ, Jung SH, Shim HJ, Bae WK, Hwang EC, et al: Class III $\beta$-tubulin is a predictive marker for taxane-based chemotherapy in recurrent and metastatic gastric cancer. BMC Cancer 13: 431 , 2013.

22. Yu J, Gao J, Lu Z, Gong J, Li Y, Dong B, Li Z, Zhang X and Shen L: Combination of microtubule associated protein-tau and $\beta$-tubulin III predicts chemosensitivity of paclitaxel in patients with advanced gastric cancer. Eur J Cancer 50: 2328-2335, 2014

23. Nitiss JL: DNA topoisomerase II and its growing repertoire of biological functions. Nat Rev Cancer 9: 327-337, 2009.

24. Dingemans AM, Witlox MA, Stallaert RA, van der Valk P, Postmus PE and Giaccone G: Expression of DNA topoisomerase IIalpha and topoisomerase IIbeta genes predicts survival and response to chemotherapy in patients with small cell lung cancer. Clin Cancer Res 5: 2048-2058, 1999.

25. Ceppi P, Longo M, Volante M, Novello S, Cappia S, Bacillo E, Selvaggi G, Saviozzi S, Calogero R, Papotti M and Scagliotti GV: Excision repair cross complementing-1 and topoisomerase IIalpha gene expression in small-cell lung cancer patients treated with platinum and etoposide: A retrospective study. J Thorac Oncol 3: 583-589, 2008.

26. Dingemans AC, van Ark-Otte J, Span S, Scagliotti GV, van der Valk P, Postmus PE and Giaccone G: Topoisomerase IIalpha and other drug resistance markers in advanced non-small cell lung cancer. Lung Cancer 32: 117-128, 2001.
27. Knoop AS, Knudsen H, Balslev E, Rasmussen BB, Overgaard J, Nielsen KV, Schonau A, Gunnarsdóttir K, Olsen KE, Mouridsen H, et al: Retrospective analysis of topoisomerase IIa amplifications and deletions as predictive markers in primary breast cancer patients randomly assigned to cyclophosphamide, methotrexate, and fluorouracil or cyclophosphamide, epirubicin, and fluorouracil: Danish breast cancer cooperative group. J Clin Oncol 23: 7483-7490, 2005

28. Li X, Liu Y, Chen W, Fang Y, Xu H, Zhu HH, Chu M, Li W, Zhuang G and Gao WQ: TOP2Ahigh is the phenotype of recurrence and metastasis whereas TOP2Aneg cells represent cancer stem cells in prostate cancer. Oncotarget 5: 9498-9513, 2014.

29. Masaoka A: Staging system of thymoma. J Thorac Oncol 5 (10 Suppl 4): S304-S312, 2010.

30. Travis W, Brambilla W, Müller-Hermelink H and Harris C: Chapter 3. Tumors of the thymus. World health organization classification of tumors. Pathology and genetics of tumors of the lung, pleura, thymus and heart. IARC press, 3rd edition, Lyon, 2004.

31. Shimizu K, Kaira K, Tomizawa Y, Sunaga N, Kawashima O, Oriuchi N, Tominaga H, Nagamori S, Kanai Y, Yamada M, et al: ASC amino-acid transporter 2 (ASCT2) as a novel prognostic marker in non-small cell lung cancer. Br J Cancer 110: 2030-2039, 2014.

32. Buck AC, Schirrmeister HH, Guhlmann CA, Diederichs CG, Shen C, Buchmann I, Kotzerke J, Birk D, Mattfeldt T and Reske SN: Ki-67 immunostaining in pancreatic cancer and chronic active pancreatitis: Does in vivo FDG uptake correlate with proliferative activity? J Nucl Med 42: 721-725, 2001.

33. Kaira K, Serizawa M, Koh Y, Miura S, Kaira R, Abe M, Nakagawa K, Ohde Y, Okumura T, Naito T, et al: Expression of excision repair cross-complementation group 1, breast cancer susceptibility 1 , and $\beta$ III-tubulin in thymic epithelial tumors. J Thorac Oncol 6: 606-613, 2011

34. McCarroll JA, Gan PP, Liu M and Kavallaris M: betaIII-tubulin is a multifunctional protein involved in drug sensitivity and tumorigenesis in non-small cell lung cancer. Cancer Res 70: 4995-5003, 2010.

35. McCarroll JA, Gan PP, Erlich RB, Liu M, Dwarte T, Sagnella SS, Akerfeldt MC, Yang L, Parker AL, Chang MH, et al: TUBB3/BIII-tubulin acts through the PTEN/AKT signaling axis to promote tumorigenesis and anoikis resistance in non-small cell lung cancer. Cancer Res 75: 415-425, 2015.

36. McCarroll JA, Sharbeen G, Liu J, Youkhana J, Goldstein D, McCarthy N, Limbri LF, Dischl D, Ceyhan GO, Erkan M, et al: BIII-tubulin: A novel mediator of chemoresistance and metastases in pancreatic cancer. Oncotarget 6: 2235-2249, 2015.

37. National Comprehensive Cancer Network (NCCN), NCCN clinical practice guidelines in oncology. Thymomas and thymic carcinomas version 1. 2015. Abstract available at www.nccn.org/professionals/physician_gls/pdf/thymic.pdf.

38. Galmarini CM, Treilleux I, Cardoso F, Bernard-Marty C, Durbecq V, Gancberg D, Bissery MC, Paesmans M, Larsimont D, Piccart MJ, et al: Class III beta-tubulin isotype predicts response in advanced breast cancer patients randomly treated either with single-agent doxorubicin or docetaxel. Clin Cancer Res 14: 4511-4516, 2008

39. Chen T, Sun Y, Ji P, Kopetz S and Zhang W: Topoisomerase IIa in chromosome instability and personalized cancer therapy. Oncogene 34: 4019-4031, 2015.

40. Burgess DJ, Doles J, Zender L, Xue W, Ma B, McCombie WR, Hannon GJ, Lowe SW and Hemann MT: Topoisomerase levels determine chemotherapy response in vitro and in vivo. Proc Natl Acad Sci USA 105: 9053-9058, 2008.

41. Desmedt C, Di Leo A, de Azambuja E, Larsimont D, Haibe-Kains B, Selleslags J, Delaloge S, Duhem C, Kains JP, Carly B, et al: Multifactorial approach to predicting resistance to anthracyclines. J Clin Oncol 29: 1578-1586, 2011.

42. Tinari N, Lattanzio R, Natoli C, Cianchetti E, Angelucci D, Ricevuto E, Ficorella C, Marchetti P, Alberti S, Piantelli M and Iacobelli S: Changes of topoisomerase IIalpha expression in breast tumors after neoadjuvant chemotherapy predicts relapse-free survival. Clin Cancer Res 12: 1501-1506, 2006.

43. Ferrandina G, Petrillo M, Carbone A, Zannoni G, Martinelli E, Prisco M,Pignata S, Breda E, Savarese A and Scambia G: Prognostic role of topoisomerase-IIalpha in advanced ovarian cancer patients. Br J Cancer 98: 1910-1915, 2008.

44. Liu JM, Wang LS, Huang MH, Hsu WH, Yen SH, Shiau CY, Li AF, Tiu CM, Tseng SW and Huang BS: Topoisomerase 2alpha plays a pivotal role in the tumor biology of stage IV thymic neoplasia. Cancer 109: 502-509, 2007. 
45. Hsu C, Kuo SH, Hu FC, Cheng AL, Shih JY, Yu CJ, Lin CC, Huang TC, Yang PC and Yang CH: Gemcitabine plus conventional-dose epirubicin versus gemcitabine plus cisplatin as first-line chemotherapy for stage IIIB/IV non-small cell lung carcinoma-a randomized phase II trial. Lung Cancer 62: 334-343, 2008.

46. Wong N, Yeo W, Wong WL, Wong NL, Chan KY, Mo FK, Koh J, Chan SL, Chan AT, Lai PB, et al: TOP2A overexpression in hepatocellular carcinoma correlates with early age onset, shorter patients survival and chemoresistance. Int J Cancer 124: 644-652, 2009.

47. Pichierri P, Franchitto A, Mosesso P, Proietti de Santis L, Balajee AS and Palitti F: Werner's syndrome lymphoblastoid cells are hypersensitive to topoisomerase II inhibitors in the G2 phase of the cell cycle. Mutat Res 459: 123-133, 2000
48. Terry SY, Riches AC and Bryant PE: Suppression of topoisomerase IIalpha expression and function in human cells decreases chromosomal radiosensitivity. Mutat Res 663: 40-45, 2009.

49. Bryant PE, Riches AC, Shovman O, Dewar JA and Adamson DJ: Topoisomerase II $\alpha$ levels and G2 radiosensitivity in T-lymphocytes of women presenting with breast cancer. Mutagenesis 27: 737-741, 2012.

50. Kim JS, Kim SY, Lee M, Kim SH, Kim SM and Kim EJ: Radioresistance in a human laryngeal squamous cell carcinoma cell line is associated with DNA methylation changes and topoisomerase II $\alpha$. Cancer Biol Ther 16: 558-566, 2015. 